\title{
Performance study of interferometric small-sats to detect exoplanets: updated exoplanet yield and application to nearby exoplanets
}

Dandumont, Colin, Kammerer, Jens, Defrère, Denis, Loicq, Jérôme

Colin Dandumont, Jens Kammerer, Denis Defrère, Jérôme Loicq, "Performance study of interferometric small-sats to detect exoplanets: updated exoplanet yield and application to nearby exoplanets," Proc. SPIE 11446, Optical and Infrared Interferometry and Imaging VII, 114462G (13 December 2020); doi: 10.1117/12.2562119 


\title{
Performance study of interferometric small-sats to detect exoplanets:Updated exoplanet yield and application to nearby exoplanets
}

\author{
Colin Dandumont ${ }^{\mathrm{a}, \mathrm{b}}$, Jens Kammerer ${ }^{\mathrm{c}, \mathrm{d}}$, Denis Defrère ${ }^{\mathrm{e}}$, and Jérôme Loicq $^{\mathrm{a}, \mathrm{b}}$ \\ ${ }^{a}$ Centre Spatial de Liège, Université de Liège, Avenue Pré-Aily, 4031 Angleur, Belgium \\ ${ }^{b}$ Space sciences, Technologies \& Astrophysics Research (STAR) Institute, University of Liege, \\ Liege, Belgium \\ 'European Southern Observatory, Karl-Schwarzschild-Straße 2, 85748 Garching, Germany \\ ${ }^{\mathrm{d}}$ Research School of Astronomy \& Astrophysics, Australian National University, ACT 2611, \\ Australia \\ ' Instituut voor Sterrenkunde, Katholieke Universiteit Leuven, Celestijnenlaan 200D, B-3001 \\ Leuven, Belgium
}

\begin{abstract}
Nulling interferometry is considered as one of the most promising solutions to spectrally characterize rocky exoplanets in the habitable zone of nearby stars. It provides both high angular resolution and starlight mitigation. It requires however several technologies that need to be demonstrated before a large interferometry space-based mission flies. A small-sat mission is a good technological precursor. Based on a Bracewell architecture, this unique satellite can demonstrate some key components (null capability, fiber injection, achromatic phase shifter). Scientific capabilities of such a mission are presented. An exoplanet detection yield is derived, and we show that the detection of exoplanets around nearby stars is feasible.
\end{abstract}

Keywords: interferometry, satellites, exoplanets, astronomy, space optics

\section{INTRODUCTION}

Since the first exoplanet discovery 25 years ago, ${ }^{1}$ more than 4200 exoplanets have been confirmed and more than 5000 are still awaiting confirmation*. These exoplanets were mostly detected by the transit and the radial velocity methods. These two methods are wonderful to detect exoplanets but the next big step is clearly to spectrally characterize a larger number of rocky planets in the habitable zone of their star to analyze their atmosphere and search for bio-signatures. However, characterizing exoplanet atmospheres is very challenging due to the high angular resolution needed and the huge star/planet contrast. Nulling interferometry is one of the most promising direct methods to tackle this challenge in the near-IR $(0.7-3 \mu \mathrm{m})$, and the mid-IR $(3-20 \mu \mathrm{m})$ domains.

Nulling interferometer is based on the idea of R.N. Bracewell. In 1978, he proposed a space-based interferometer with two apertures and a recombination of light in phase opposition. ${ }^{2}$ It produces a dark fringe on the line-of-sight and the stellar emission is strongly suppressed. For an off-axis source, the light can be transmitted depending on the baseline length and the wavelength, which define the interferometer transmission map. Even if this technique is currently used on the ground, e.g. with the Large Binocular Telescope Interferometer ${ }^{3,4}$ or the Guided-Light Interferometric Nulling Technology, ${ }^{5,6}$ a space-based mission is needed to avoid atmospheric effects (turbulence, opacity at specific wavelengths). ${ }^{7}$ The Large Interferometer for Exoplanets (LIFE) mission project is fitted for this task. Its goal is to characterize the atmosphere of dozens of terrestrial planets at the mid-infrared

Further author information: (Send correspondence to C. Dandumont)

E-mail: colin.dandumont@uliege.be

${ }^{*}$ https://exoplanets.nasa.gov, November 30, 2020

Optical and Infrared Interferometry and Imaging VII, edited by Peter G. Tuthill, Antoine Mérand,

Stephanie Sallum, Proc. of SPIE Vol. 11446, 114462G · C 2020 SPIE

CCC code: $0277-786 X / 20 / \$ 21 \cdot$ doi: $10.1117 / 12.2562119$

Proc. of SPIE Vol. 11446 114462G-1 
wavelengths and assess their potential habitability. ${ }^{8}$ Some spectral bands contain several molecular absorption features (e.g. $\left.\mathrm{H}_{2} \mathrm{O}, \mathrm{CO}_{2}, \mathrm{O}_{3}, \mathrm{CH}_{4}\right) .{ }^{9}$ Depending on the waveband, one can measure the planet temperature (mid-IR) or measure the planet radius (mid-IR) or a combination of the radius and the albedo (visible - NIR). The planetary orbital parameters can be derived whatever the spectral band. ${ }^{10}$

The present proceeding is dedicated to the analysis of the scientific return of a smaller mission. As nulling interferometry was never achieved in space, a smaller space-based mission can be considered as a useful prerequisite to demonstrate some key components before developing an L-class mission, such as LIFE. ${ }^{11,12}$ In Ref. 13, our previous work, four configurations were considered, from small to medium satellites. The main objective was to develop our tools and present our first analyzes. Based on a planet population synthesis tool, we showed that, even without platform stability constraints, CubeSats can hardly detect giant exoplanets. A PROBA-like mission could detect more than 120 putative exoplanets and a more ambitious mission such as FKSI could detect 250 exoplanets. One of our conclusions is that small platforms are well suited to test and validate critical technological components needed for a larger mission as well as perform scientific observations. ${ }^{13}$

In this paper, only small missions from Ref. 13 are considered: two CubeSats and one larger spacecraft of the size of the ESA PROBA-class family. All of them are in a Bracewell configuration, e.g. with only two apertures with recombination in the pupil plane. Thanks to the planet population synthesis tool (P-POP) developed by J. Kammerer \& S.P. Quanz (2018), ${ }^{14}$ synthetic planet populations, based on the Kepler satellite data, are generated around the same sample of nearby main-sequence stars (distance $<20 \mathrm{pc}$ ) as in Ref. 13 (326 stars). From these populations, detection yields are computed. In this proceeding, the goal is to derive requirements on the optical train temperature, the optical path difference (OPD), and the relative tip/tilt angle. We present some wavelength dependency that was not shown previously. We also present in Sec. 4 detection capabilities for nearby stars. The 28 nearest known exoplanets were selected and for each configuration, the detection yield is derived. The impact of the optical train temperature, the optical path difference (OPD), and the relative tip/tilt angle is also discussed.

\section{SATELLITE CONFIGURATIONS}

As stated above, only the three small configurations are considered (Tab. 1). For each of them, a pupil diameter and a baseline length are chosen. The impact of the optical chain temperature, optical path difference (OPD), and the relative tip/tilt angle is discussed in Sec. 3 and 4. All considered satellites are a fiber-coupled Bracewell interferometer. A simplified optical block diagram is available in Ref. 13. The elements in the optical path are two telescopes, mounted on the same structure and spaced by the baseline length, tip/tilt mirrors to correct any errors, optical delay lines to correct the optical path differences (OPD), a fiber injection system to filter the signal, an achromatic phase shifter ( $\pi$ delay in one arm), the beam combiner to receive the light from both apertures and create interference in the pupil plane, and two photodiodes, one at the constructive output and one at the destructive output.

\begin{tabular}{l|l|l|l} 
& \multicolumn{1}{|c|}{ CubeSat 6U } & CubeSat 12U & PROBA-size \\
\hline Size & $0.6 \times 0.1 \times 0.1 \mathrm{~m}$ & $1.1 \times 0.1 \times 0.1 \mathrm{~m}$ & $1 \mathrm{~m}^{3}$ \\
\hline Baseline length & $0.5 \mathrm{~m}$ & $1 \mathrm{~m}$ & $5 \mathrm{~m}$ \\
\hline Pupil diameter & $0.08 \mathrm{~m}$ & $0.08 \mathrm{~m}$ & $0.25 \mathrm{~m}$ \\
\hline \multicolumn{2}{r}{ Table 1. Three small-sat configurations considered in this study. }
\end{tabular}

\subsection{CubeSats}

CubeSats are part of the "NewSpace" trend, and thanks to the miniaturization of all platform components, it becomes reasonable to think about astronomical CubeSats. Science CubeSats are more and more a reality and they can now achieve high-performance science missions. They can not of course replace larger missions. ${ }^{15}$ ASTERIA (Arcsecond Space Telescope Enabling Research in Astrophysics) from the Jet Propulsion Laboratory (JPL) is one of the best known science CubeSat. It was able to acquire photometric information of the transiting super-Earth 55 Cancri e. ${ }^{16}$ 
CubeSats are defined by their number of units. One unit, or $1 \mathrm{U}$, corresponds to $0.1 \times 0.1 \times 0.1 \mathrm{~m}$. In this article, we consider two Cubesat concepts, a $6 \mathrm{U}$ and a $12 \mathrm{U}$. $6 \mathrm{U}$ CubeSats are officially defined as $2 \times 3 \times 1 \mathrm{U}^{\ddagger}$ but an alternative exists. Although rarely used, Nanoracks, who deploys CubeSats from the International Space Station, offers the possibility to launch a $6 \mathrm{U}$ CubeSat with a linear form factor $(6 \times 1 \times 1 \mathrm{U}) .{ }^{\S}$ This choice will increase the baseline length of the satellite and so the angular resolution of the interferometer. For the $12 \mathrm{U}$ CubeSat, the idea is the same. Unfortunately, to our knowledge, no deployer for $12 \mathrm{U}$ CubeSats with a linear form factor exists. Therefore, we considered a typical $12 \mathrm{U}$ Cubesat $(2 \times 3 \times 2 \mathrm{U})$ with a deployable mechanism to obtain a $12 \times 1 \mathrm{U}$ Cubesat. In these configurations, both Cubesats have a maximized baseline length. Due to size constraints, the size of their primary mirrors is limited to $8 \mathrm{~cm}$.

\subsection{PROBA-size}

The PROBA-size satellite is based on the PROBA family (Project for On-Board Autonomy) from ESA. It is a satellite class designed around innovation at a reduced cost. Three successful missions are currently flying (PROBA-1 since 2001, PROBA-2 since 2009, and PROBA-V since 2013). The next one, PROBA-3 is planned to be launched soon. Composed of two spacecraft, these satellites will maintain formation autonomously to submillimeter precision in the lateral plane and millimeter precision in the longitudinal plane at distances of $150 \mathrm{~m}$ or more. ${ }^{17}$ It is an important step towards a large interferometric mission since formation flying is necessary to increase the baseline length up to a few hundred meters. ${ }^{7}$ The size of all PROBA satellites is around $1 \mathrm{~m}^{3}$. With deployable arms, one can assume to achieve a baseline length up to $5 \mathrm{~m}$.

\section{EXOPLANET MISSIONS YIELD}

In this section, three types of missions are considered (Tab. 1). The P-POP algorithm, described below, is used to generate a set of synthetic planets around 326 real nearby main-sequence stars (distance $<20 \mathrm{pc}$ ), the same as in Ref. 13. Depending on their characteristics (size, orbit, star), our algorithm computes the integration time needed to reach a Signal-to-Noise Ratio of 5 (see below). If this threshold is reached before $24 \mathrm{~h}$ of observation, the planet is considered as detected.

With this detection yield, some intrinsic characteristics of each configuration can be analyzed. Here are reviewed the optical chain temperature, the optical path difference, and the tip/tilt accuracy as a function of the operating wavelength.

\subsection{Monte-Carlo simulation tool: P-POP}

The Kepler satellite, from NASA, discovered more than half of the current exoplanet population thanks to the transit method. ${ }^{18}$ Occurrence rates of exoplanets, based on their size, orbital period, and stellar host, type were derived. These occurence rates are aggregated in the SAG13 model from NASA. ${ }^{\mathbb{I}}$ From these, we generate synthetic planet populations using the Monte-Carlo tool (P-POP) described in Kammerer \& Quanz (2018) around 326 real main-sequence stars located within a radius of $20 \mathrm{pc}$ around the Earth. ${ }^{14,19}$ Around each star, we draw 100 planetary populations (or universe) from our occurrence rate distribution, yielding a statistically robust sample of $\approx 86,000$ planets in total. Some stars can have multiple planets, whereas some have no planet. The total number of synthetic planets in a planet radius - stellar insolation plot is shown in Fig. 1. The mean value is shown as well as its standard deviation. This standard deviation is around 10\%. It is related to error of a Poisson-like distribution and is coherent with 100 trials. P-POP generates mainly terrestrial planets (below $4 \mathrm{R}_{\oplus}$ ) with stellar insolation (normalized) close to that of the Earth. This kind of planet is expected to be the most common. ${ }^{20}$

\footnotetext{
${ }^{\ddagger}$ https://www. cubesat.org/resources, November 30, 2020

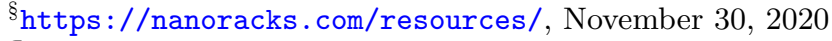

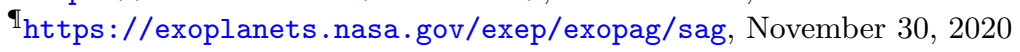



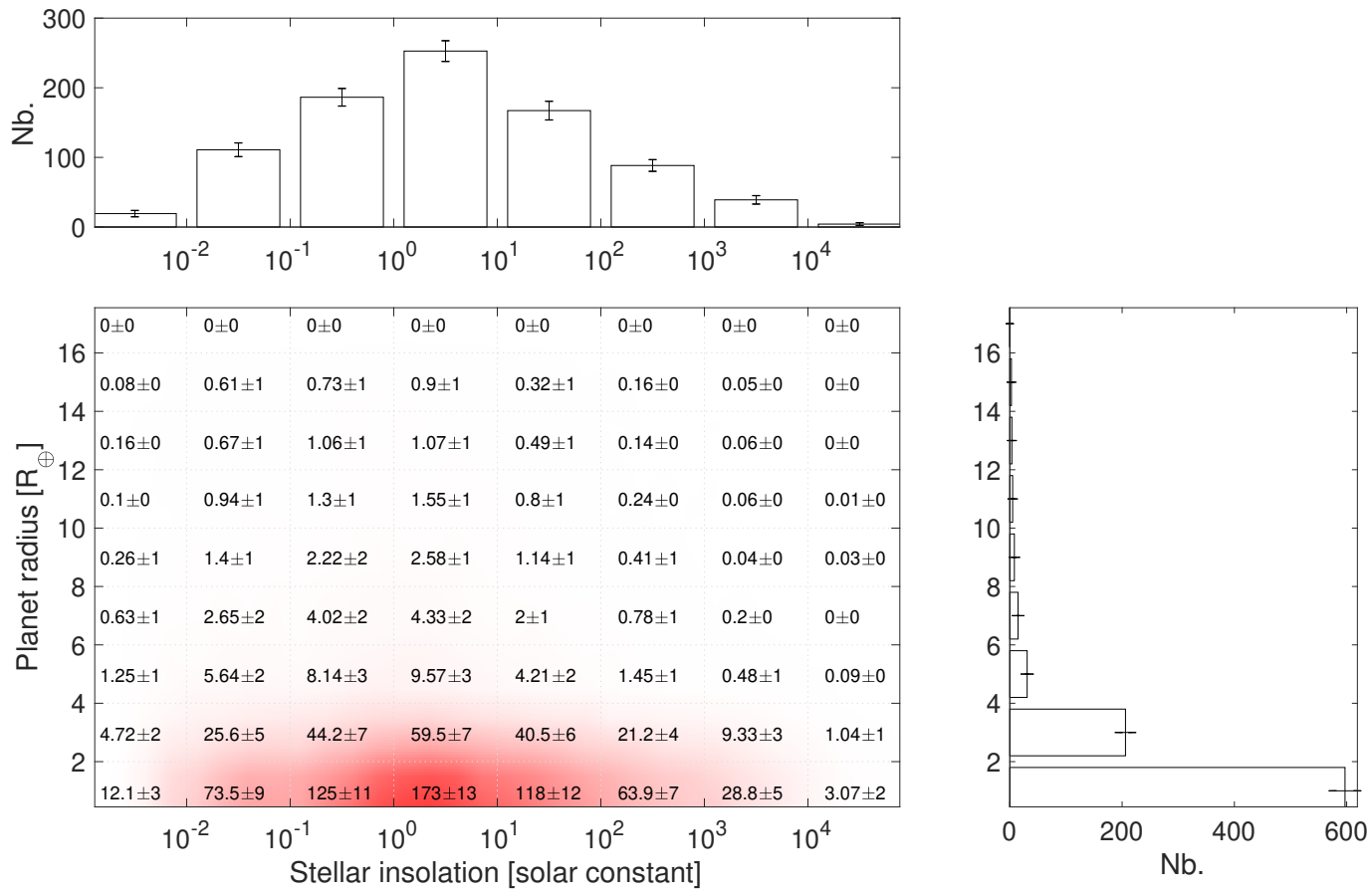

Figure 1. Mean generated P-POP population (100 trials around 326 real stars) and corresponding standard deviation. Redder (darker) region corresponds to more occurrence. Each histogram is the sum of the synthetic planets in the columns or lines of the central figure.

\subsection{Radiometric budget}

In Ref. 13, a full and detailed description of the radiometric budget is available. The integration time is derived for each generated exoplanet. As incoming fluxes are considered:

- Stellar and planetary fluxes. Stars and planets are assumed to emit as black bodies. The full exoplanet spectral density flux is the sum of two contributions: the reflected flux, which is a fraction of the emitted flux of the host star, and the thermal flux, which is directly related to the equilibrium temperature of the planet. $^{21}$

- Local zodiacal emission. Local zodiacal dust is located between the orbit of Mercury and Jupiter. It scatters sunlight at every wavelength while it emits mainly in the mid-IR (thermal radiation). We assume that this dust has a temperature of $300 \mathrm{~K}$ in the habitable zone of the Solar system.

- Exozodiacal emission. An exozodiacal disk is the extrasolar countepart of the zodiacal disk. The presence of an exozodiacal disk, depending on its inclination and emission, creates a flux that could be higher than the planetary signal. $^{22,23}$ Recent results from the Large Binocular Telescope Interferometer (LBTI) suggest the majority of Sun-like stars have relatively low HZ dust levels (best-fit median: 3 zodis, $1 \sigma$ upper limit: 9 zodis, $95 \%$ confidence: 27 zodis based on $\mathrm{N}$ band measurements; 1 zodi corresponds to the level of the zodiacal emission in the solar system), while $20 \%$ are significantly more dusty. ${ }^{24}$ In our study, the planet population synthesis tool generates an exozodiacal level based on the measured LBTI statistics.

- Instrumental background flux. The Sun and the Earth (in low Earth orbit) radiate energy towards the satellite. The baffle of each aperture, as well as the attitude of the satellite, will prevent any direct solar light from entering the interferometer. We assume that the telescopes and optical train radiate as a grey body with an emissivity of 0.25 . 


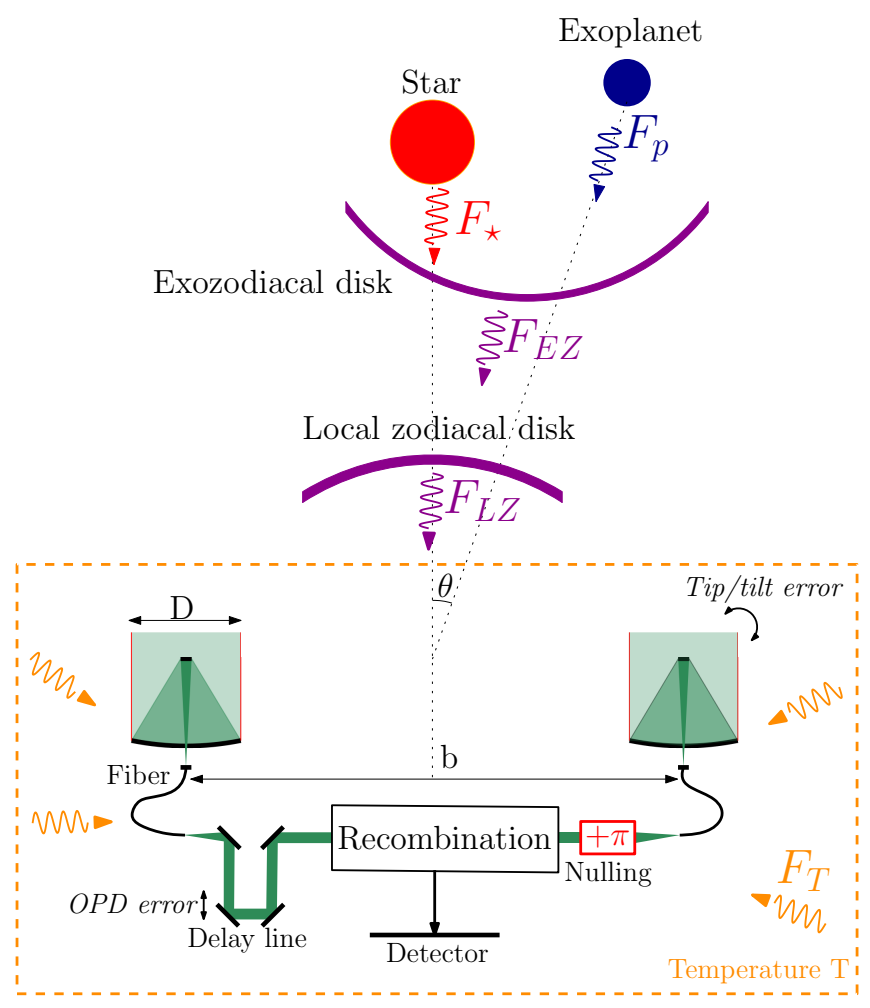

Figure 2. Schematic presentation of a Bracewell nulling interferometer, represented with the main noise sources used in our radiometric budget. $F_{*}$ - the stellar flux; $F_{p}$ - the planetary flux; $F_{E Z}$ - the exozodiacal flux; $F_{L Z}-$ the zodiacal flux; $F_{\mathrm{T}}$ - the thermal flux of the instrument (temperature T)

- Instrumental Throughput. The instrumental throughput takes into account the reflectance of the mirrors of each aperture, the fiber coupling efficiency, and the quantum efficiency of the detector. Optical fibers were not present in the Bracewell proposal, but their addition leads to the correction of phase defects of the incoming wavefront, except a relative piston. ${ }^{2,25}$ The detectors used for this application are two photodiodes. Unfortunately, the detector quantum efficiency is not constant over a large bandwidth since it depends on the detector technology. To keep a pure photonic case, specific detector properties are not considered. However, we assume a generic value of the quantum efficiency, $Q E$, of $80 \%$ not to overestimate our results.

All these contributions are represented in Fig. 2.

To compute the Signal-to-Noise Ratio (SNR), a bandwidth is necessary. As in Ref. 13, a resolution parameter, $\mathrm{R}$, of $1.2(=\lambda / \Delta \lambda)$ is selected. It corresponds to a decrease by $\approx 50 \%$ of the fiber coupling efficiency (chromatic effect). We are interested here in planet detections and not a full characterization of the planet.

\subsubsection{Null depth}

The null depth is defined as

$$
\mathrm{N}=\frac{I_{-}}{I_{+}}
$$

where $I_{+}$is the intensity at the constructive output and $I_{-}$at the destructive one. ${ }^{26}$ It is one of the main figures of merit of nulling interferometry. This null depth can be decomposed as a time-averaged null depth, $\overline{\mathrm{N}}$, in the presence of active OPD matching and intensity balancing, and a root-mean-square (RMS) fluctuation of the null level, $\sigma_{\mathrm{N}}{ }^{26}$ 
The time-averaged null depth of a Bracewell interferometer observing a single star is given by

$$
\overline{\mathrm{N}}=\frac{1}{4}\left[\sigma_{\phi}^{2}+\frac{\pi^{2}}{4}\left(\frac{\theta_{\mathrm{dia}}}{\lambda_{\mathrm{sh}} / b}\right)^{2}+\sigma_{I}^{2}\right]
$$

if the contribution from polarization is neglected and the phase dispersion across the passband is considered constant in vacuum. ${ }^{26} \sigma_{\phi}^{2}$ is the variance of the phase error. The second term, $\frac{\pi^{2}}{4}\left(\frac{\theta_{\mathrm{dia}}}{\lambda_{\mathrm{sh}} / b}\right)^{2}$, is related to the finite extent of the star and leads to a "stellar leakage" in the destructive output with $\theta_{\text {dia }}$ the star angular diameter, $b$, the baseline length and $\lambda_{\mathrm{sh}}$, the shortest wavelength in the bandwidth. $\sigma_{I}^{2}$ is the variance of the fractional intensity deviations. ${ }^{26}$ This equation contributes to the mean residual stellar flux. The RMS fluctuation of the null level is given by ${ }^{26}$

$$
\sigma_{\mathrm{N}}=\sqrt{\frac{\sigma_{\phi}^{4}+\sigma_{I}^{4}}{8}} .
$$

Impact of $\sigma_{\phi}^{2}$ and $\sigma_{I}^{2}$ parameters are discussed below and requirements on these values are derived. Thanks to the single-mode optical fiber, any wavefront errors are converted into intensity errors, $\sigma_{I}$, at the output, which are less severe than a pure phase effect on the nulling. Photometric imbalance from the stellar leaks (residual stellar flux) is of the second order as compared with phase aberrations. ${ }^{25}$

\subsubsection{Integration time}

To retrieve the planet signal from all signals and distinguish it from the background, a solution is to rotate the interferometer around its line-of-sight as originally proposed by Bracewell. ${ }^{2}$ The planet crosses bright and dark fringes, and its flux is modulated. The background, considered as uniform, is not modulated. This modulation comes nevertheless with a drawback. The measured planetary flux is, on average, reduced to $50 \%$ in the case of a Bracewell interferometer.

To retrieve the modulated planet signal, the minimal requested Signal-to-Noise Ratio (SNR) is fixed to 5 . During the acquisition, the spacecraft performs a whole number of rotations. This SNR is expressed per second and leads to the computation of the integration time. If the orbital parameters of the planet are known a priori (from indirect methods), one can compute the total integration time to get a requested Signal-to-Noise Ratio, $\mathrm{SNR}_{\text {req }}$, of 5 . In the case of an unknown planet, one needs to fix a maximum allowed integration time. It is fixed by the architecture constraints as well as the platform stability in time. The SNR generated during 1 second, $\mathrm{SNR}_{1 \mathrm{sec}}$, is given by

$$
\mathrm{SNR}_{1 \mathrm{sec}}=\frac{\mathrm{OPF}}{\sqrt{\left(N_{s}^{2}+N_{\text {inst }}^{2}\right)}}
$$

where OPF is the output planet flux (planet flux multiplied by the instrumental throughput and the transmission map), $N_{s}$ the shot noise and, $N_{i n s t}$ is the instrumental stellar leakage. This last is the multiplication of the $\sigma_{\mathrm{N}}$ term (Eq. 3) by the output stellar flux (remaining stellar flux). It is a noise directly linked to the stability of the null and how it can be controlled. It depends on the co-phasing error (OPD) and the mismatches of intensities (tip/tilt) at the beam recombination.

The integration time, $t_{i}$, for a minimal required $\mathrm{SNR}\left(\mathrm{SNR}_{\text {req }}\right)$ is simply equal to

$$
t_{i}=\left(\frac{\mathrm{SNR}_{\mathrm{req}}}{\mathrm{SNR}_{1 \mathrm{sec}}}\right)^{2}=\left(\frac{5}{\mathrm{SNR}_{1 \mathrm{sec}}}\right)^{2} .
$$

\subsection{Instrumental temperature effect}

As shown in Ref. 13, the instrumental temperature or the optical chain temperature is the main noise contributor at long wavelength. It drastically limits the number of detected planets. In this proceeding, we, therefore, limit our investigation up to $7.5 \mu \mathrm{m}$ as the number of detections is very low at longer wavelengths. Figure 3 shows, for the three configurations, the number of detected exoplanets as a function of the operating wavelength and the instrumental temperature. Exoplanets are considered as detected if the SNR is at least 5 after $24 \mathrm{~h}$ of observation. 

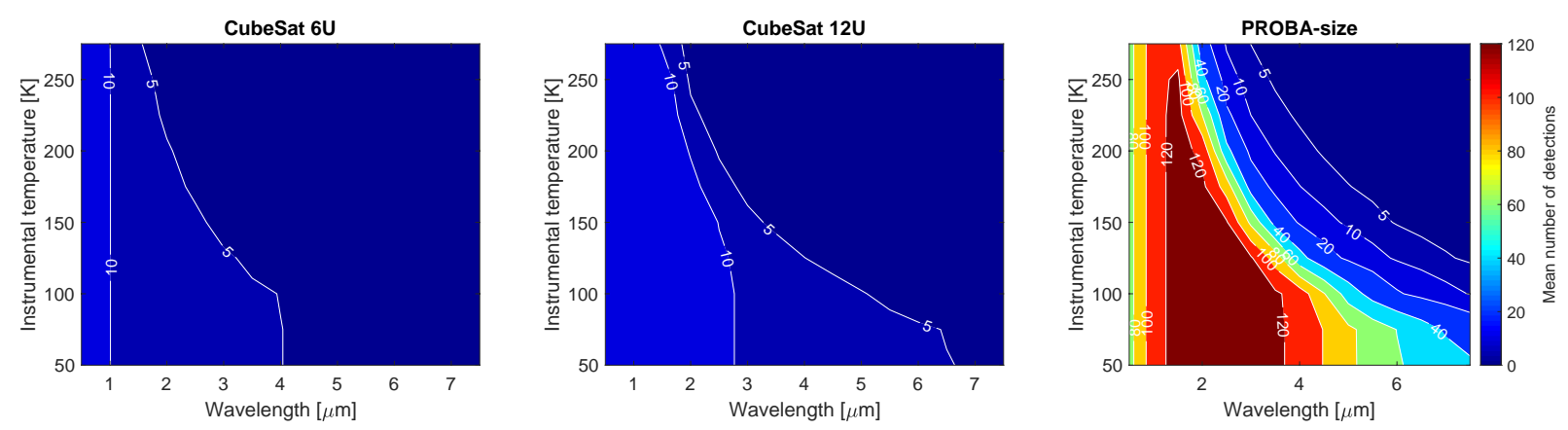

Figure 3. Mean number of detected planets with the three configurations defined in Tab. 1 as a function of the operating wavelength (resolution parameter, $R=1.2$ ) and the instrumental temperature. A planet is considered as detected if the SNR is a least 5 with an integration time of $24 \mathrm{~h}$. Platform stability constraints (OPD and tip/tilt) are not considered yet. They are respectively considered in Sec. 3.4 and 3.5.

Note that this figure can not lead to the direct determination of the best operating waveband. Indeed, for each wavelength represented, a bandwidth is already considered as previously discussed $(\mathrm{R}=1.2)$.

CubeSats can hardly detect exoplanets ( 10 over $\approx 870$ generated exoplanets) while the PROBA-size is well suited for this purpose with more than 120 potential exoplanet detections. The instrumental temperature has a very limited impact in the $0.5-3.0 \mu \mathrm{m}$ range. Even at $250 \mathrm{~K}$, it is not the main noise contributor. As expected, if we go to the infrared domain $(>3.5 \mu \mathrm{m})$, the instrumental temperature flux begins to dominate and the number of detections decreases for all three configurations. As the number of detections is higher in the beginning, the PROBA-size configuration is however less affected.

From Fig. 3, we can put some requirements on the instrumental temperature to achieve for each configuration. These temperatures are used in the following. For both CubeSats, $200 \mathrm{~K}$ is selected. The PROBA satellite needs to be more cooled to detect around 120 exoplanets. $150 \mathrm{~K}$ is selected. It is less restrictive than in Ref. 13 (100 K was used as assumption) as this new representation shows that the instrumental temperature has less impact in short wavelength.

\subsection{Impact of the optical path difference}

The optical path difference (OPD) is another figure of merit of a nulling interferometer. There is always a residual OPD. It is a pure piston that is not corrected by the single-mode waveguide. Figure 4 represents how this residual OPD and the wavelength affect the number of detections. This residual OPD has a very large impact. Less than $5 \mathrm{~nm}$ are allowed for the PROBA-size configuration to avoid any large drop of detections. For CubeSats, any large deviation will lead to a non-detection scenario.

The wavelength-dependency can also be seen. It comes directly from Eq. 2, with the term $\sigma_{\phi}^{2}$. It equals $\left(2 \pi \sigma_{\mathrm{OPD}} / \lambda\right)^{2}$ where $\sigma_{\mathrm{OPD}}$ is the value represented in Fig. 4 .

\subsection{Impact of tip/tilt}

In this section, we are interested in the RMS differential tip/tilt. It means that the satellite has a zero-mean tip/tilt angle but both pupils are misaligned by an angle, the RMS differential tip/tilt. This misalignment, which can be static or dynamic (temperature, vibration, etc.) leads to intensity imbalance at the output of the fibers and degradation of the time-average null depth as shown in Eq. 2 ( $\sigma_{I}$ term).

Figure 5 represents the number of detections as a function of the wavelength and the RMS differential tip/tilt. The requirements need to be very stringent. More than 50 mas leads already to a drop of detections for the PROBA-size configuration. 

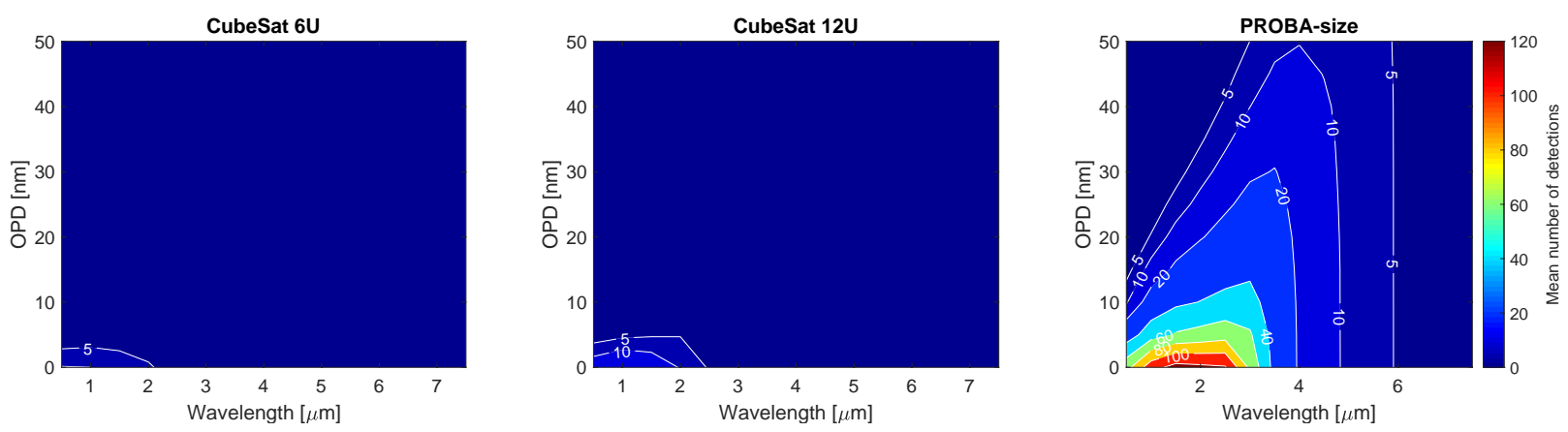

Figure 4. Mean number of detected planets with the three configurations defined in Tab. 1 as a function of the operating wavelength (resolution parameter, $R=1.2$ ) and the residual optical path difference. A planet is considered as detected if the SNR is a least 5 with an integration time of $24 \mathrm{~h}$. Instrumental temperature: $200 \mathrm{~K}$ for both CubeSats and $150 \mathrm{~K}$ for PROBA. No tip/tilt considered.
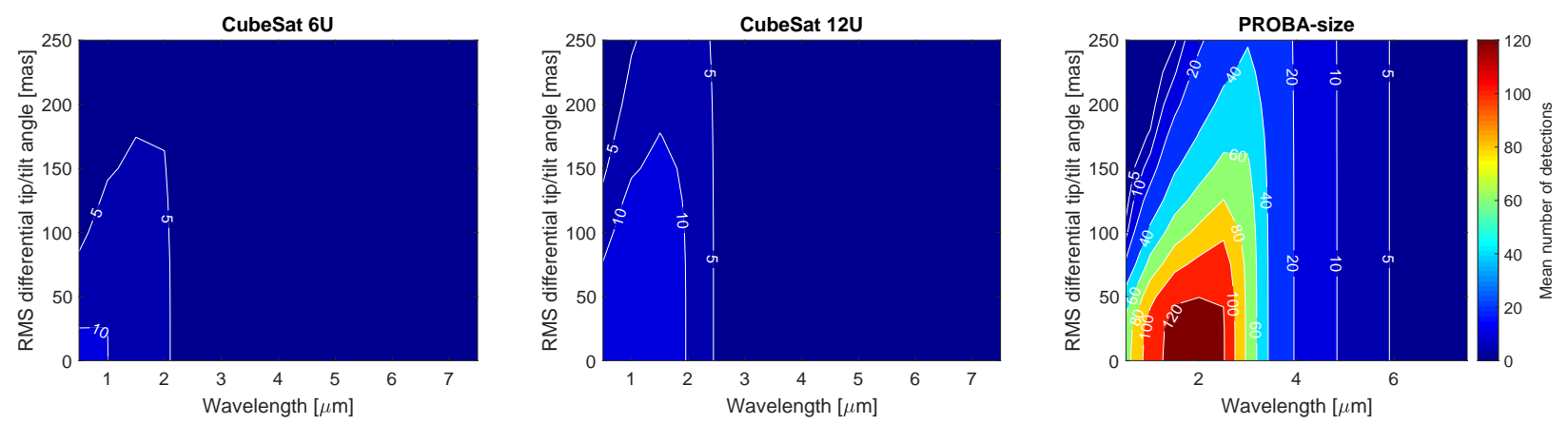

Figure 5. Number of detected planets with the three configurations defined in Tab. 1 as a function of the operating wavelength (resolution parameter, $R=1.2$ ) and the RMS differential tip/tilt. A planet is considered as detected if the $\mathrm{SNR}$ is a least 5 with an integration time of $24 \mathrm{~h}$. Instrumental temperature: $200 \mathrm{~K}$ for both CubeSats and $150 \mathrm{~K}$ for PROBA. No OPD considered.

\section{YIELD FOR NEARBY EXOPLANETS}

In this section, we are interested in real nearby exoplanets. It allows us to have information about the detectability and characterization of known exoplanets. As above, instrumental temperature, OPD and, tip/tilt effects are analyzed.

\subsection{Nearby exoplanets}

From exoplanet.eu, we have selected the 28 nearest exoplanets. We have not considered two brown dwarfs (Luhman $16 \mathrm{~A}$ and Luhman $16 \mathrm{~B}$ ) and one sub-brown dwarf (WISE 0855-0714). Table 3 in Appendix A regroups all stellar and planets properties. All these planets lie in the vicinity of the Earth $(<4.0 \mathrm{pc})$ and were all discovered by the radial velocity method.

\subsection{Instrumental temperature effect}

Figure 6 show how many planets out of the 28 each configuration can detect. It is interesting to show that the CubeSat $6 \mathrm{U}$ can not detect any of these exoplanets. The CubeSat $12 \mathrm{U}$ can only detect GJ $887 \mathrm{c}$, which is large a sub-Neptune ( $\left.3.16 R_{\text {Earth }}\right)$. The integration time treshold is set to $24 \mathrm{~h}$ but it does not have an influence on both CubeSats. In best conditions, the CubeSat $6 \mathrm{U}$ needs to integrate during $69 \mathrm{~h}$ (parameters: $\lambda=1 \mu \mathrm{m} / T=50 \mathrm{~K}$ ) to detect only one planet, GJ $887 \mathrm{c}$. To detect Proxima b, the nearest exoplanet, the CubeSat $6 \mathrm{U}$ needs to integrate during $1852 \mathrm{~h}(1 \mathrm{\mu m} / 50 \mathrm{~K})$, the CubeSat $12 \mathrm{U} 187 \mathrm{~h}(1 \mathrm{\mu m} / 50 \mathrm{~K})$. The PROBA-size spacecraft detects it in $2.2 \mathrm{~h}(3 \mu \mathrm{m} / 50 \mathrm{~K})$, without considering any platform stability constraints. 

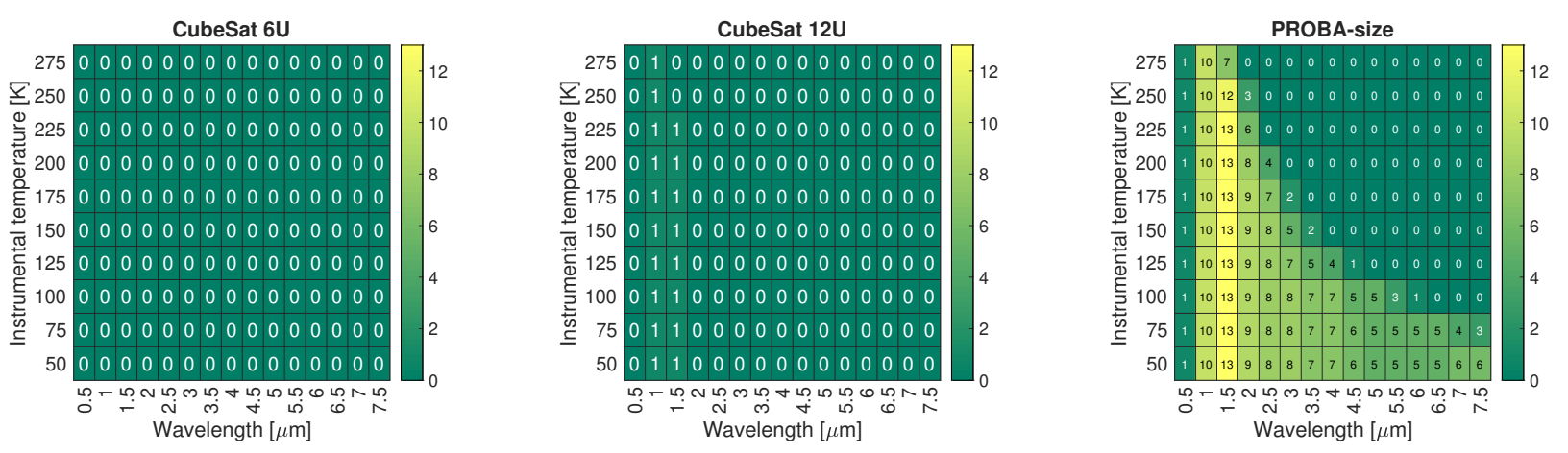

Figure 6. Number of detected planets out of the 28 nearest exoplanets (Tab. 3) with the three configurations defined in Tab. 1 as a function of the operating wavelength (resolution parameter, $R=1.2$ ) and the instrumental temperature. A planet is considered as detected if the SNR is a least 5 with an integration time of $24 \mathrm{~h}$. Platform stability constraints (tip/tilt and OPD) are not considered yet. They are considered in Sec. 4.3.

\begin{tabular}{|c|c|c|c||c|c|c|c|}
\hline & \multicolumn{3}{|c|}{ Integration time $(\mathrm{h})$} & \multicolumn{3}{c|}{ Integration time (h) } \\
\hline & CubeSat 6U & CubeSat 12U & PROBA-size & & CubeSat 6U & CubeSat 12U & PROBA-size \\
\hline Proxima Centauri b & $3 \mathrm{e}+03$ & 273 & 12 & eps Ind A b & Inf & Inf & Inf \\
\hline Proxima Centauri c & $2 \mathrm{e}+05$ & $3 \mathrm{e}+05$ & Inf & tau Cet e & $7 \mathrm{e}+02$ & $2 \mathrm{e}+03$ & $9 \mathrm{e}+03$ \\
\hline Barnard's star b & $1 \mathrm{e}+04$ & $3 \mathrm{e}+04$ & $1 \mathrm{e}+04$ & tau Cet f & $3 \mathrm{e}+04$ & $1 \mathrm{e}+05$ & $3 \mathrm{e}+05$ \\
\hline Wolf 359 b & $1 \mathrm{e}+06$ & $1 \mathrm{e}+06$ & $3 \mathrm{e}+06$ & tau Cet g & $1 \mathrm{e}+03$ & 264 & 76 \\
\hline Wolf 359 c & $1 \mathrm{e}+05$ & $7 \mathrm{e}+03$ & 1 & tau Cet h & $1 \mathrm{e}+03$ & 375 & $1 \mathrm{e}+03$ \\
\hline Lalande 21185 b & $1 \mathrm{e}+03$ & 149 & 10 & GJ 1061 b & $8 \mathrm{e}+06$ & $5 \mathrm{e}+05$ & 18 \\
\hline eps Eridani b & $1 \mathrm{e}+03$ & $4 \mathrm{e}+03$ & Inf & GJ 1061 c & $6 \mathrm{e}+06$ & $4 \mathrm{e}+05$ & 16 \\
\hline GJ 887 b & $2 \mathrm{e}+03$ & 249 & 3 & GJ 1061 d & $7 \mathrm{e}+06$ & $4 \mathrm{e}+05$ & 26 \\
\hline GJ 887 c & 152 & 19 & 3 & GJ 273 b & $1 \mathrm{e}+05$ & $8 \mathrm{e}+03$ & 17 \\
\hline Ross 128 b & $9 \mathrm{e}+05$ & $6 \mathrm{e}+04$ & 10 & GJ 273 c & $4 \mathrm{e}+05$ & $3 \mathrm{e}+04$ & 10 \\
\hline GJ 15A b & $2 \mathrm{e}+04$ & $2 \mathrm{e}+03$ & 5 & GJ 273 d & $9 \mathrm{e}+03$ & $2 \mathrm{e}+04$ & $4 \mathrm{e}+03$ \\
\hline YZ Cet b & $9 \mathrm{e}+06$ & $6 \mathrm{e}+05$ & 29 & GJ 273 e & $2 \mathrm{e}+04$ & $5 \mathrm{e}+04$ & $9 \mathrm{e}+03$ \\
\hline YZ Cet c & $5 \mathrm{e}+06$ & $3 \mathrm{e}+05$ & 16 & Teegarden's star b & $2 \mathrm{e}+08$ & $1 \mathrm{e}+07$ & 290 \\
\hline YZ Cet d & $5 \mathrm{e}+06$ & $3 \mathrm{e}+05$ & 18 & Teegarden's star c & $2 \mathrm{e}+08$ & $1 \mathrm{e}+07$ & 343 \\
\hline
\end{tabular}

Table 2. Integration time for each configuration (cf. Tab. 1). $\lambda=1.5 \mu \mathrm{m}$ and $T=150 \mathrm{~K}$. Platform stability constraints (tip/tilt and OPD) are not considered. Green cell represent planets that are detected in less than $24 \mathrm{~h}$ of integration time.

The PROBA-concept is not able to detect all of the 28 nearest exoplanets, even with small instrumental temperature. In fact, at some wavelengths, mainly lower ones, some planets are too distant from their host star and are out of outer working angle $(0.514 \lambda / D)$. Figure 7 represents the contrast as a function of angular separation at $1.5 \mathrm{\mu m}$, the optimum for the PROBA-size case (cf. Fig. 6). The inner working angle (IWA - $\lambda / 2 B$ ) is represented for each configuration. It represents the first maximum in the transmission map. Therefore, the transmission is not zero on the left side but is drastically reduced. It explains why some planets (GJ $887 \mathrm{~b} / \mathrm{c}$ for instance) can be detected with the PROBA-size case. Their lower contrast allows a detection, especially since no platform stability constraints are considered at this stage. For planets beyond the outer working angle (OWA - $0.514 \lambda / D)$, the transmission is zero and therefore the integration time is infinite.

Table 2 gives the integration time for each exoplanet at $1.5 \mu \mathrm{m}$ and $150 \mathrm{~K}$. This temperature is a good compromise for the PROBA-size satellite. Above this value, as shown in Fig. 6, one needs to stick around $1.5 \mathrm{~m}$. Below, it will be aggressive constraints for a satellite in low Earth orbit. These results are consistent with the one derived from P-POP in Sec. 3.

\subsection{Impact of the optical path difference and the tip/tilt}

Results presented above are quite optimistic since both residual optical path difference and RMS differential tip/tilt angle were neglected. Figure 8 shows how these two instrumental have a drastic effect on detections.

\footnotetext{
"Wolf 359 c has a non-zero integration time in Tab. 2 even if in Fig 7 this planet is outside of the OWA. It is due to the spectral bandwidth: $1.5 \mathrm{\mu m}$ is the mid-band value. At the upper edge of the band, the planet is inside the OWA and so detectable.
} 


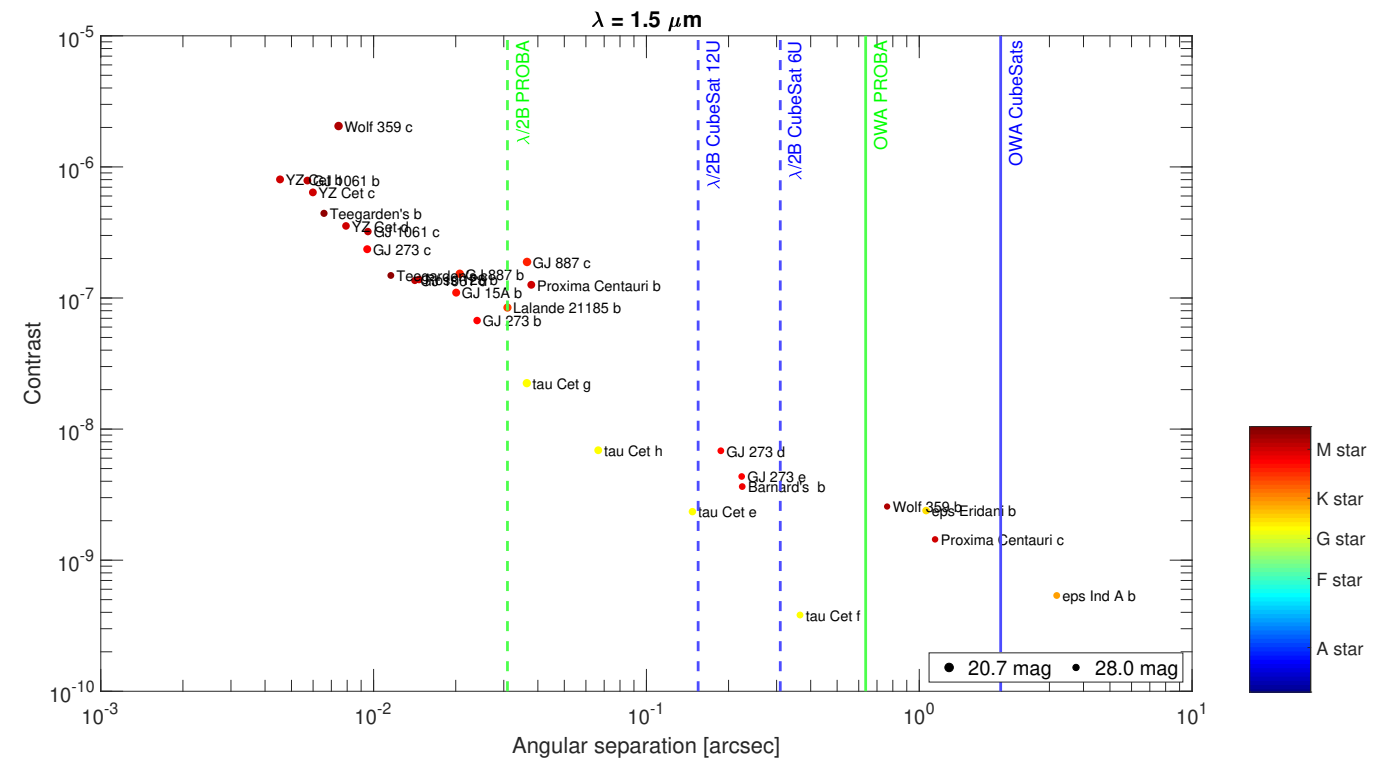

Figure 7. Contrast at $1.5 \mu \mathrm{m}$ of the 28 nearest exoplanets as a function of their maximum angular separation. The spectral type of the host star is represented in color. The size of the bubble is related to the magnitude of the exoplanet. The Inner Working Angle (IWA) is the first maximum in the transmission map and is equal to IWA $=\lambda / 2 B$. The Outer Working Angle (OWA) is the transmission limit of the interferometer and is equal to OWA $=0.514 \lambda / D$.
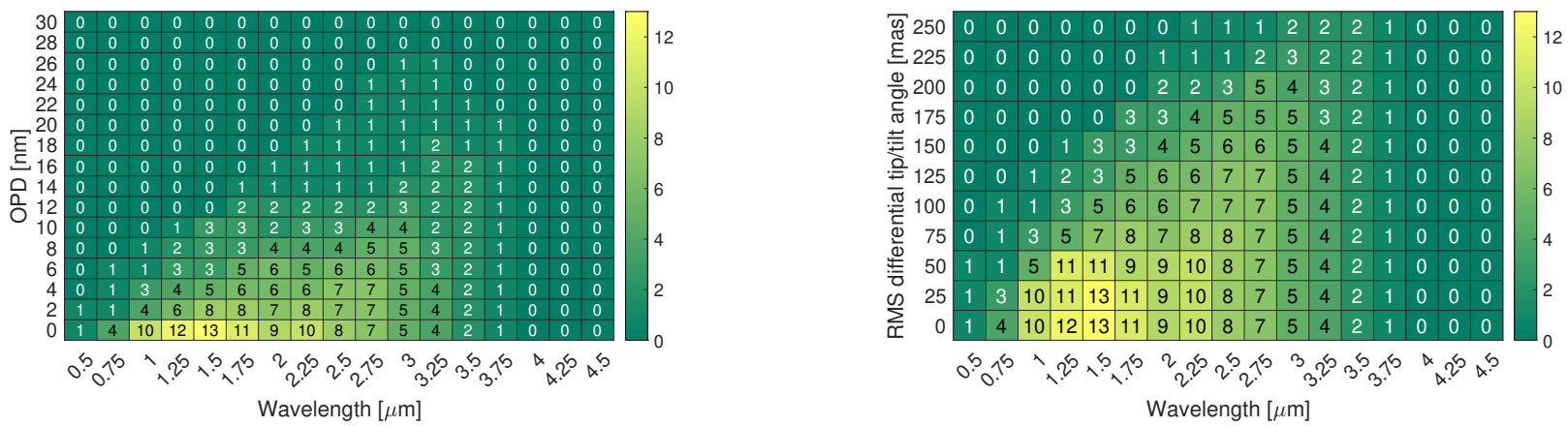

Figure 8. Number of detected planets out of the 28 nearest exoplanets (Tab. 3) with the three configurations defined in Tab. 1 as a function of the operating wavelength (resolution parameter, $R=1.2$ ) and (left) the residual optical path difference and (right) the RMS differential tip/tilt. A planet is considered as detected if the SNR is a least 5 with an integration time of $24 \mathrm{~h}$.

Since both CubeSats can hardly detect exoplanets, only the PROBA-size case is analyzed.

To detect near exoplanets, constraints are though and only $2 \mathrm{~nm}$ of residual optical path difference are sufficient to lost half of the detection at $1.5 \mu \mathrm{m}$. The pattern in Fig. 8 (left) is the same as the one in Fig 4 with the wavelength dependency explicitly shown. This wavelength-dependency also exists with the RMS differential tip/tilt. As previously derived, with P-POP, above $50 \mathrm{~nm}$, the number of detections starts to decrease to only half of the maximum detection value.

\section{CONCLUSIONS}

In this proceeding, we derived an exoplanet detection yield based on a state-of-the-art planet population synthesis tool (P-POP) for three small satellite configurations. The effect of the instrumental temperature, as well as two 
major instrumental effects (optical path difference and tip/tilt angle), are discussed. Then, the 28 nearest exoplanets are analyzed with the same procedure.

The instrumental temperature at constant operating wavelength has a lower impact has previously thought. If only nearby stars are considered, in the $1.5-2 \mu \mathrm{m}$ region, an optical train around $200 \mathrm{~K}$ is sufficient to reduce the thermal contribution. However, as shown for a large sample of stars thanks to P-POP, the temperature needs to be lower $(150 \mathrm{~K})$ to keep the maximum of detections. Constraints are also strong on instrumental stability. Only a few nm of OPD is sufficient to drastically decrease the number of detections, for nearby stars or the large sample. It will be a real challenge for a space-based interferometer to achieve these values.

This work also shows that a CubeSat configuration has very little chance to detect known exoplanets in the vicinity of the Earth. To detect exoplanets with such a small platform, a large sample of stars is needed. With the 326 real main-sequence stars used with P-POP, only a few fractions of their exoplanets (5-10 exoplanets) can be detected. If the goal is to characterize at least several exoplanets, a larger mission, as a PROBA-one, is needed. It will avoid any uncertainties and will detect exoplanets with more confidence.

Modelisation of OPD and tip/tilt control loops as well as a full optical layout are the next step to characterize this PROBA configuration and improve our results and detection yield.

\section{APPENDIX A. NEARBY EXOPLANETS TABLE}

Table 3 shows all the stellar and planetary information of the 28 nearest exoplanets used in this study.

\begin{tabular}{|c|c|c|c|c|c|c|c|c|c|c|}
\hline \multicolumn{6}{|c|}{ EXOPLANETS } & \multicolumn{5}{|c|}{ STAR } \\
\hline Name & $\begin{array}{c}\text { Mass } \\
\left(M_{\text {Earth }}\right)\end{array}$ & $\begin{array}{c}\text { Mass*sin(i) } \\
\left(M_{\text {Earth }}\right)\end{array}$ & $\begin{array}{l}\text { Radius } \\
\left(R_{\text {Earth }}\right)\end{array}$ & $\begin{array}{c}\text { Semi-major axis } \\
\text { (AU) }\end{array}$ & $\begin{array}{c}\text { Temperature } \\
(\mathrm{K})\end{array}$ & Name & $\begin{array}{c}\text { Distance } \\
(\mathrm{pc})\end{array}$ & $\begin{array}{l}\text { Radius } \\
\left(R_{\text {Sun }}\right)\end{array}$ & Spectral type & $\begin{array}{c}\text { Temperature } \\
(\mathrm{K})\end{array}$ \\
\hline Proxima Centauri b & & 1.2 & 1.1 & 0.05 & 216 & Proxima Centauri & 1.3 & 0.1 & M5.5V & 3050 \\
\hline Proxima Centauri c & 9.5 & & 3.4 & 1.5 & 39 & Proxima Centauri & 1.3 & 0.1 & M5.5V & 3050 \\
\hline Barnard's star b & & 3.2 & 1.5 & $0.4^{2}$ & 105 & Barnard's star & 1.8 & 0.2 & M4V & 3278 \\
\hline Wolf $359 \mathrm{~b}$ & & 43.9 & 5.7 & 1.8 & $36^{1}$ & Wolf 359 & 2.4 & $0.2^{3}$ & M6V & $2800^{4}$ \\
\hline Wolf $359 \mathrm{c}$ & & 3.8 & 1.6 & 0.02 & $368^{1}$ & Wolf 359 & 2.4 & $0.2^{3}$ & $\mathrm{M} 6 \mathrm{~V}$ & $2800^{4}$ \\
\hline Lalande 21185 b & & 2.7 & 1.4 & 0.08 & 370 & Lalande 21185 & 2.6 & 0.4 & M1.5V & 3828 \\
\hline eps Eridani b & 244.8 & & 10.0 & 3.4 & $116^{1}$ & eps Eridani & 3.2 & 0.9 & $\mathrm{~K} 2 \mathrm{~V}$ & 5116 \\
\hline GJ 887 b & & 4.2 & 1.6 & 0.07 & 468 & GJ 887 & 3.3 & 0.5 & M1V & 3688 \\
\hline GJ $887 \mathrm{c}$ & & 7.6 & 3.2 & 0.1 & 352 & GJ 887 & 3.3 & 0.5 & M1V & 3688 \\
\hline Ross $128 \mathrm{~b}$ & & 1.4 & 1.1 & 0.05 & 256 & Ross 128 & 3.6 & 0.2 & M4 & 3192 \\
\hline GJ $15 \mathrm{~A} \mathrm{~b}$ & & 3.0 & 1.4 & 0.07 & $364^{1}$ & GJ $15 \mathrm{~A}$ & 3.6 & 0.4 & $\mathrm{M} 2 \mathrm{~V}$ & 3567 \\
\hline YZ Cet b & & 0.7 & 0.9 & 0.02 & $432^{1}$ & YZ Cet & 3.6 & 0.2 & M4.5 & 3056 \\
\hline YZ Cet c & & 1.1 & 1.0 & 0.02 & $376^{1}$ & YZ Cet & 3.6 & 0.2 & M4.5 & 3056 \\
\hline YZ Cet d & & 1.1 & 1.0 & 0.03 & 327 & YZ Cet & 3.6 & 0.2 & M4.5 & 3056 \\
\hline eps Ind $\mathrm{A} \mathrm{b}$ & & 1033.0 & 16.2 & 11.6 & 51 & eps Ind A & 3.6 & $0.7^{5}$ & $\mathrm{~K} 2 \mathrm{~V}$ & $4630^{5}$ \\
\hline tau Cet e & & 4.0 & 1.6 & 0.5 & $286^{1}$ & tau Cet & 3.7 & 0.8 & G8.5V & 5344 \\
\hline $\operatorname{tau}$ Cet $\mathrm{f}$ & & 4.0 & 1.6 & 1.3 & $182^{1}$ & tau Cet & 3.7 & 0.8 & G8.5V & 5344 \\
\hline tau Cet g & & 1.8 & 1.2 & 0.1 & $576^{1}$ & tau Cet & 3.7 & 0.8 & G8.5V & 5344 \\
\hline tau Cet h & & 1.8 & 1.2 & 0.2 & $426^{1}$ & tau Cet & 3.7 & 0.8 & G8.5V & 5344 \\
\hline GJ $1061 \mathrm{~b}$ & & 1.4 & 1.1 & 0.02 & $355^{1}$ & GJ 1061 & 3.7 & 0.2 & M5.5V & 2953 \\
\hline GJ $1061 \mathrm{c}$ & & 1.7 & 1.2 & 0.04 & $275^{1}$ & GJ 1061 & 3.7 & 0.2 & M5.5V & 2953 \\
\hline GJ $1061 \mathrm{~d}$ & & 1.6 & 1.2 & 0.05 & $226^{1}$ & GJ 1061 & 3.7 & 0.2 & M5.5V & 2953 \\
\hline GJ $273 \mathrm{~b}$ & 3.0 & & 1.4 & 0.1 & $268^{1}$ & GJ 273 & 3.8 & 0.3 & M3.5 & 3382 \\
\hline GJ $273 \mathrm{c}$ & 1.2 & & 1.1 & 0.04 & $426^{1}$ & GJ 273 & 3.8 & 0.3 & M3.5 & 3382 \\
\hline GJ $273 \mathrm{~d}$ & 11.0 & & 3.6 & 0.7 & $96^{1}$ & GJ 273 & 3.8 & 0.3 & M3.5 & 3382 \\
\hline GJ $273 \mathrm{e}$ & 9.4 & & 3.4 & 0.8 & $88^{1}$ & GJ 273 & 3.8 & 0.3 & M3.5 & 3382 \\
\hline Teegarden's star b & & 1.0 & 1.0 & 0.03 & $240^{1}$ & Teegarden's & 3.8 & 0.107 & $\mathrm{M} 7.0 \mathrm{~V}$ & $2637^{6}$ \\
\hline Teegarden's star c & & 1.1 & 1.0 & 0.04 & $181^{1}$ & Teegarden's star & 3.8 & 0.1 & M7.0V & $2637^{6}$ \\
\hline
\end{tabular}

Table 3. Stellar and planetary information of the 28 nearest exoplanets. Data, unless specified, are from exoplanet.eu. Inclinations and eccentricities are not considered. Planets are assumed to be a their maximum elongation. Albedo is assumed at 0.3 on the full spectral range. All radius are derived from the mass, considering the Earth density (< 7 MEarth) or the Jupiter density (Mass > 7 MEarth).

${ }^{1}$ When not available, the effective temperature is computed assuming equilibrium (Pin = Pout) and the Stefan-Boltzmann law.

${ }^{2}$ From 27.

${ }^{3}$ From 28.

${ }^{4}$ From 29.

${ }^{5}$ From 30.

${ }^{6}$ From 31.

\section{ACKNOWLEDGMENTS}

The authors acknowledge the financial support of the University of Liège, the Centre Spatial de Liège. The research leading to these results has received funding from the European Union's Horizon 2020 research and 
innovation programme under Grant Agreement 730890 (OPTICON). D.D. acknowledges funding from the European Research Council (grant agreement 866070).

\section{REFERENCES}

[1] Mayor, M. and Queloz, D., "A Jupiter-mass companion to a solar-type star," Nature 378, 355-359 (Nov. 1995). [doi:10.1038/378355a0].

[2] Bracewell, R. N., "Detecting nonsolar planets by spinning infrared interferometer," Nature 274(5673), 780 (1978). [doi:10.1038/274780a0].

[3] Hinz, P. M., Angel, J. R. P., Woolf, N. J., Hoffmann, W. F., and McCarthy, D. W., "Imaging Extra-solar Systems from the Ground: The MMT and LBT Nulling Interferometers," Working on the Fringe: Optical and IR Interferometry from Ground and Space 194, 401 (1999).

[4] Defrère, D., Hinz, P., Skemer, A., Bailey, V., Downey, E., Durney, O., Eisner, J., Hill, J. M., Hoffmann, W. F., Leisenring, J., McMahon, T., Montoya, M., Spalding, E., Stone, J., Vaz, A., Absil, O., Esposito, S., Kenworthy, M., Mennesson, B., Millan-Gabet, R., Nelson, M., Puglisi, A., Skrutskie, M. F., and Wilson, J., "Exoplanet science with the LBTI: instrument status and plans," in [Techniques and Instrumentation for Detection of Exoplanets VII], 9605, 96051G, International Society for Optics and Photonics (Sept. 2015). [doi:10.1117/12.2188912].

[5] Lagadec, T., Norris, B., Gross, S., Arriola, A., Gretzinger, T., Cvetojevic, N., Lawrence, J., Withford, M., and Tuthill, P., "GLINT South: a photonic nulling interferometer pathfinder at the Anglo-Australian Telescope for high contrast imaging of substellar companions," in [Optical and Infrared Interferometry and Imaging VI], Creech-Eakman, M. J., Tuthill, P. G., and Mérand, A., eds., 10701, 238 - 245, International Society for Optics and Photonics, SPIE (2018).

[6] Norris, B. R. M., Cvetojevic, N., Lagadec, T., Jovanovic, N., Gross, S., Arriola, A., Gretzinger, T., Martinod, M.-A., Guyon, O., Lozi, J., Withford, M. J., Lawrence, J. S., and Tuthill, P., "First on-sky demonstration of an integrated-photonic nulling interferometer: the GLINT instrument," Monthly Notices of the Royal Astronomical Society 491, 4180-4193 (11 2019).

[7] Defrère, D., Léger, A., Absil, O., Beichman, C., Biller, B., Danchi, W. C., Ergenzinger, K., Eiroa, C., Ertel, S., Fridlund, M., Muñoz, A. G., Gillon, M., Glasse, A., Godolt, M., Grenfell, J. L., Kraus, S., Labadie, L., Lacour, S., Liseau, R., Martin, G., Mennesson, B., Micela, G., Minardi, S., Quanz, S. P., Rauer, H., Rinehart, S., Santos, N. C., Selsis, F., Surdej, J., Tian, F., Villaver, E., Wheatley, P. J., and Wyatt, M., "Space-based infrared interferometry to study exoplanetary atmospheres," Experimental Astronomy 46, 543-560 (Dec 2018). [doi:10.1007/s10686-018-9613-2].

[8] Quanz, S. P., Kammerer, J., Defrère, D., Absil, O., Glauser, A. M., and Kitzmann, D., "Exoplanet science with a space-based mid-infrared nulling interferometer," in [Optical and Infrared Interferometry and Imaging VI], 10701, 107011I, International Society for Optics and Photonics (July 2018). [doi:10.1117/12.2312051].

[9] des Marais, D. J., Harwit, M. O., Jucks, K. W., Kasting, J. F., Lin, D. N., Lunine, J. I., Schneider, J., Seager, S., Traub, W. A., and Woolf, N. J., "Remote Sensing of Planetary Properties and Biosignatures on Extrasolar Terrestrial Planets," Astrobiology 2(2) (2002). [doi:10.1089/15311070260192246].

[10] Beichman, C., Fridlund, M., Traub, W., Stapelfeldt, K., Quirrenbach, A., and Seager, S., "Comparative planetology and the search for life beyond the solar system," Protostars and Planets 5, 915-928 (2007). [doi:10.1089/15311070260192246].

[11] Monnier, J., Aarnio, A., Absil, O., Anugu, N., Baines, E., Bayo, A., Berger, J.-P., Cleeves, L. I., Dale, D., Danchi, W., de Wit, W. J., Defrère, D., Domagal-Goldman, S., Elvis, M., Froebrich, D., Gai, M., Gandhi, P., Garcia, P., Gardner, T., Gies, D., Gonzalez, J.-F., Gunter, B., Hoenig, S., Ireland, M., Jorgensen, A. M., Kishimoto, M., Klarmann, L., Kloppenborg, B., Kluska, J., Knight, J. S., Kral, Q., Kraus, S., Labadie, L., Lawson, P., Le Bouquin, J.-B., Leisawitz, D., Lightsey, E. G., Linz, H., Lipscy, S., MacGregor, M., Matsuo, H., Mennesson, B., Meyer, M., Michael, E. A., Millour, F., Mozurkewich, D., Norris, R., Ollivier, M., Packham, C., Petrov, R., Pueyo, L., Pope, B., Quanz, S., Ragland, S., Rau, G., Regaly, Z., Riva, A., Roettenbacher, R., Savini, G., Setterholm, B., Sewilo, M., Smith, M., Spencer, L., ten Brummelaar, T., Turner, N., van Belle, G., Weigelt, G., and Wittkowski, M., "A Realistic Roadmap to Formation Flying Space Interferometry," in [Bulletin of the American Astronomical Society], 51, 153 (Sept. 2019). 
[12] Rinehart, S. A., Savini, G., Holland, W., Absil, O., Defrère, D., Spencer, L., Leisawitz, D., Rizzo, M., Juanola-Paramon, R., and Mozurkewich, D., "The path to interferometry in space," in [Optical and Infrared Interferometry and Imaging V], Malbet, F., Creech-Eakman, M. J., and Tuthill, P. G., eds., 9907, 187 201, International Society for Optics and Photonics, SPIE (2016).

[13] Dandumont, C., Defrère, D., Kammerer, J., Absil, O., Quanz, S. P., and Loicq, J., "Exoplanet detection yield of a space-based Bracewell interferometer from small to medium satellites," Journal of Astronomical Telescopes, Instruments, and Systems 6(3), 1 - 20 (2020).

[14] Kammerer, J. and Quanz, S. P., "Simulating the exoplanet yield of a space-based mid-infrared interferometer based on Kepler statistics," Astronomy \&3 Astrophysics 609, A4 (2018). [doi:10.1051/0004-6361/201731254].

[15] Liddle, J. D., Holt, A. P., Jason, S. J., Donnell, K. A. O., and Stevens, E. J., "Space science with CubeSats and nanosatellites," Nature Astronomy 4(November) (2020).

[16] Knapp, M., Seager, S., Demory, B.-O., Krishnamurthy, A., Smith, M. W., Pong, C. M., Bailey, V. P., Donner, A., Pasquale, P. D., Campuzano, B., Smith, C., Luu, J., Babuscia, A., Bocchino, Jr., R. L., Loveland, J., Colley, C., Gedenk, T., Kulkarni, T., Hughes, K., White, M., Krajewski, J., and Fesq, L., "Demonstrating High-precision Photometry with a CubeSat: ASTERIA Observations of 55 Cancri e," AJ 160(1), 23 (2020).

[17] Casti, M., Bemporad, A., Fineschi, S., Capobianco, G., Loreggia, D., Noce, V., Landini, F., Thizy, C., Galano, D., and Rougeot, R., "PROBA-3 formation-flying metrology: algorithms for the shadow position sensor system," in [International Conference on Space Optics - ICSO 2018], Karafolas, N., Sodnik, Z., and Cugny, B., eds., 289, SPIE, Chania, Greece (July 2019). [doi:10.1117/12.2536209].

[18] Borucki, W. J., Koch, D., Basri, G., Batalha, N., Brown, T., Caldwell, D., Caldwell, J., ChristensenDalsgaard, J., Cochran, W. D., DeVore, E., Dunham, E. W., Dupree, A. K., Gautier, T. N., Geary, J. C., Gilliland, R., Gould, A., Howell, S. B., Jenkins, J. M., Kondo, Y., Latham, D. W., Marcy, G. W., Meibom, S., Kjeldsen, H., Lissauer, J. J., Monet, D. G., Morrison, D., Sasselov, D., Tarter, J., Boss, A., Brownlee, D., Owen, T., Buzasi, D., Charbonneau, D., Doyle, L., Fortney, J., Ford, E. B., Holman, M. J., Seager, S., Steffen, J. H., Welsh, W. F., Rowe, J., Anderson, H., Buchhave, L., Ciardi, D., Walkowicz, L., Sherry, W., Horch, E., Isaacson, H., Everett, M. E., Fischer, D., Torres, G., Johnson, J. A., Endl, M., MacQueen, P., Bryson, S. T., Dotson, J., Haas, M., Kolodziejczak, J., Van Cleve, J., Chandrasekaran, H., Twicken, J. D., Quintana, E. V., Clarke, B. D., Allen, C., Li, J., Wu, H., Tenenbaum, P., Verner, E., Bruhweiler, F., Barnes, J., and Prsa, A., "Kepler Planet-Detection Mission: Introduction and First Results," Science 327(5968), 977-980 (2010). [doi:10.1126/science.1185402].

[19] Quanz, S. P., Crossfield, I., Meyer, M. R., Schmalzl, E., and Held, J., "Direct detection of exoplanets in the 3-10 $\mu \mathrm{m}$ range with E-ELT/METIS," International Journal of Astrobiology 14(02), 279-289 (2015). [doi:10.1017/S1473550414000135].

[20] Mulders, G. D., "Planet Populations as a Function of Stellar Properties," in [Handbook of Exoplanets], Deeg, H. J. and Belmonte, J. A., eds., 2009-2034, Springer International Publishing, Cham (2018). [doi:10.1007/978-3-319-55333-7_153].

[21] Defrère, D., Absil, O., Hanot, C., and Fridlund, M., "Potential of space-based infrared Bracewell interferometers for planet detection," Techniques and Instrumentation for Detection of Exoplanets III 6693 (2007). [doi:10.1117/12.758669].

[22] Defrère, D., Absil, O., Den Hartog, R., Hanot, C., and Stark, C., "Nulling interferometry: impact of exozodiacal clouds on the performance of future life-finding space missions," Astronomy and Astrophysics 509, A9 (Jan. 2010). [doi:10.1051/0004-6361/200912973].

[23] Roberge, A., Chen, C. H., Millan-Gabet, R., Weinberger, A. J., Hinz, P. M., Stapelfeldt, K. R., Absil, O., Kuchner, M. J., and Bryden, G., "The Exozodiacal Dust Problem for Direct Observations of Exo-Earths," Publications of the Astronomical Society of the Pacific 124, 799-808 (Aug. 2012). [doi:10.1086/667218].

[24] Ertel, S., Defrère, D., Hinz, P., Mennesson, B., Kennedy, G. M., Danchi, W. C., Gelino, C., Hill, J. M., Hoffmann, W. F., Mazoyer, J., et al., "The hosts survey for exozodiacal dust: observational results from the complete survey," The Astronomical Journal 159(4), 177 (2020).

[25] Mennesson, B., Ollivier, M., and Ruilier, C., "Use of single-mode waveguides to correct the optical defects of a nulling interferometer," Journal of the Optical Society of America A 19(3), 596 (2002). [doi:10.1364/JOSAA.19.000596]. 
[26] Serabyn, E., "Nulling interferometry: symmetry requirements and experimental results," 328 (2000). [doi:10.1117/12.390223].

[27] Ribas, I., Tuomi, M., Reiners, A., Butler, R. P., Morales, J. C., Perger, M., Dreizler, S., Rodríguez-López, C., González Hernández, J. I., Rosich, A., Feng, F., Trifonov, T., Vogt, S. S., Caballero, J. A., Hatzes, A., Herrero, E., Jeffers, S. V., Lafarga, M., Murgas, F., Nelson, R. P., Rodríguez, E., Strachan, J. B., TalOr, L., Teske, J., Toledo-Padrón, B., Zechmeister, M., Quirrenbach, A., Amado, P. J., Azzaro, M., Béjar, V. J., Barnes, J. R., Berdiñas, Z. M., Burt, J., Coleman, G., Cortés-Contreras, M., Crane, J., Engle, S. G., Guinan, E. F., Haswell, C. A., Henning, T., Holden, B., Jenkins, J., Jones, H. R., Kaminski, A., Kiraga, M., Kürster, M., Lee, M. H., López-González, M. J., Montes, D., Morin, J., Ofir, A., Pallé, E., Rebolo, R., Reffert, S., Schweitzer, A., Seifert, W., Shectman, S. A., Staab, D., Street, R. A., Suárez Mascareño, A., Tsapras, Y., Wang, S. X., and Anglada-Escudé, G., "A candidate super-Earth planet orbiting near the snow line of Barnard's star," Nature 563(7731), 365-368 (2018).

[28] DOYLE, J. and BUTLER, C., "Optical and infrared photometry of dwarf M and K stars," Astronomy and astrophysics (Berlin. Print) 235(1), 335-339 (1990).

[29] Pavlenko, Y. V., Jones, H. R., Lyubchik, Y., Tennyson, J., and Pinfield, D. J., "Spectral energy distribution for GJ406," Astronomy and Astrophysics 447(2), 709-717 (2006).

[30] Demory, B. O., Ségransan, D., Forveille, T., Queloz, D., Beuzit, J. L., Delfosse, X., Di Folco, E., Kervella, P., Le Bouquin, J. B., Perrier, C., Benisty, M., Duvert, G., Hofmann, K. H., Lopez, B., and Petrov, R., "Massradius relation of low and very low-mass stars revisited with the VLTI," Astronomy and Astrophysics 505(1), 205-215 (2009).

[31] Rojas-Ayala, B., Covey, K. R., Muirhead, P. S., and Lloyd, J. P., "Metallicity and temperature indicators in Mdwarf K-band spectra: Testing new and updated calibrations with observations of 133 solar neighborhood Mdwarfs," Astrophysical Journal 748(2) (2012). 